\title{
Personzentrierte Beratung - Möglichkeiten in neuen Tätigkeitsfeldern
}

\author{
Helmut E. Lück • Wolfgang Rechtien • Gabriela Sewz
}

Beratung - ein unscharf konturierter Begriff und ein schwer zu umreißendes Tätigkeitsfeld: Was zunächst als Nachteil erscheint bietet jedoch auch Vorteile, nämlich die Möglichkeit und die Herausforderung zur Fundierung differenter Beratungsansätze und zur Einbindung solcher Verfahren in unterschiedliche Tätigkeitsfelder. Innerhalb dieser Formen und Formate nimmt die personzentrierte Beratung eine zunehmend bedeutsame Rolle ein, sowohl im Hinblick auf die Herausbildung von Beratung als eigenständiger Profession als auch als spezifische Kompetenz im Rahmen angrenzender Professionen.

Diesen Möglichkeiten und auch den Grenzen personzentrierter Beratung in sehr verschiedenen, zum Teil völlig neuen Beratungsfeldern widmet sich der Thementeil dieses Heftes. Bei den Aufsätzen handelt es sich ausschließlich um Zusammenfassungen von Masterarbeiten aus dem Weiterbildenden Masterstudiengang Personzentrierte Beratung (Abschluss: Master of Counselling), der am Kurt Lewin Institut für Psychologie der FernUniversität in Kooperation mit der Gesellschaft für wissenschaftliche Gesprächspsychotherapie und Beratung $(\mathrm{GwG})$ durchgeführt wurde. Teilnehmerinnen und Teilnehmer waren Angehörige beratender Berufsfelder mit einem akademischen Grad, in der Regel in einem sozialwissenschaftlichen Studiengang. Wir Editoren dieses Thementeils waren mit der Planung und Durchführung des Studiengangs an der FernUniversität befasst und haben diese Masterarbeiten betreut. Die Autorinnen und Autoren der folgenden Beiträge sind also in sehr verschiedenen Berufsfeldern beratend tätig und beziehen in ihre Aufsätze ihre spezifischen beruflichen Erfahrungen ein.

Dies gilt gleich für den ersten Beitrag von Ursula Kersting-Otte über Personzentrierte Beratung minderjähriger Frauen in der Schwangerschaft und im Schwangerschaftskonflikt. Nach Rogers sind die personzentrierte Therapie und Beratung nicht scharf voneinan-

\footnotetext{
Online publiziert: 18.01 .2011

(C) VS Verlag für Sozialwissenschaften 2011

H. E. Lück $(\bowtie) \cdot$ W. Rechtien · G. Sewz

Hagen, Deutschland

E-Mail: helmut.lueck@fernuni-hagen.de

W. Rechtien

E-Mail: wolfgang.rechtien@fernuni-hagen.de

G. Sewz

E-Mail: gabi.sewz@fernuni-hagen.de
} 
der geschieden. Stets gilt es, drei Haltungen zu verwirklichen: Empathie, Wertschätzung und Kongruenz. Personzentrierte Beratung minderjähriger Frauen erfordert und ermöglicht ein Beratungsangebot mit diesen Haltungen für minderjährige Schwangere. Sie kann so diese jungen Frauen darin unterstützen, eine tragfähige Entscheidung zu treffen und ihren Lebensentwurf einschließlich Elternschaft oder einen Gegenentwurf nach einem Schwangerschaftsabbruch zu entwickeln.

Spezifische Beratung für Frauen ist heute verbreitet. Weniger im öffentlichen Bewusstsein ist spezifische Männerberatung, mit der sich Dirk Belau befasst. Da wichtige gesellschaftliche Werte und Ordnungsprinzipien mit der männlichen Geschlechterrolle übereinstimmen (Androzentrismus), werden spezifische Männersorgen - auch von ihnen selber - wenig wahrgenommen. Dies erschwert Beratung. Belau beschreibt geschlechtsbedingte Gefühlsarmut von Männern und wie Beratung ihr begegnen kann. Er zeigt ferner, dass der personzentrierte Ansatz durch seine Betonung der Dimension „Rigidität“ (Rogers) in der Inkongruenz des Klienten den Blick auch für soziale Rangunterschiede im männlichen Beratungsbedarf öffnet. - Über die Benennung der auf Rogers zurückgehenden Beratungsform kann man streiten. Dirk Belau spricht von personenzentrierter Beratung. Inzwischen hat sich für den Ansatz von Rogers im Deutschen aber die Bezeichnung personzentriert durchgesetzt und in der Literatur findet sich beispielsweise zum Coaching die Bezeichnung ,personenzentrierte Beratung“ als Charakterisierung eines Beratungsformates und grenzt dieses ohne Bezug auf einen bestimmten Beratungsansatz ab gegenüber Team- oder Organisationsberatung. Personzentriert meint demgegenüber eine Beratungsform, die sich auf Entwicklungsprozesse der Person zentriert - in Abgrenzung zu problemzentrierter oder lösungsorientierter Beratung. So gesehen ist die Unterscheidung zwischen personenzentriert und personzentriert von mehr als nebensächlicher Bedeutung.

Die gruppenbezogene Beratung stellt in der Zahnmedizin ein eher ungewöhnliches Thema dar. Selbstverständlich sind Beratungen in der zahnärztlichen Praxis ein integraler Bestandteil der Tätigkeit. Dabei handelt es sich jedoch fast ausschließlich um Einzelberatungen der Patienten, die allenfalls um einen Lebenspartner oder einen Erziehungsberechtigten ergänzt werden. Zudem ist eine solche Beratung praktisch immer als eine medizinische Empfehlung anzusehen, die der Ratsuchende von einem Experten erhält. Doch spielen auch in der Zahnmedizin und speziell im Bereich der chronischen orofazialen Schmerzen neben somatischen auch psychische und soziale Faktoren eine wesentliche Rolle. Hier scheinen die üblichen, medizinischen Vorgehensweisen insbesondere mit Blick auf die sogenannte „Compliance“ allzu häufig nicht zu funktionieren. Aus diesem Grund stellt dieser Beitrag von Markus Fussenegger dar, warum und unter welchen Bedingungen der personzentrierte Ansatz im Allgemeinen und die Personzentrierte, gruppenbezogene Beratung im Speziellen bei diesem Klientel eine Alternative für eine erfolgreiche Beratung und Therapie darstellt.

Das Kinder- und Jugendtelefon (KJT) ist seit über 30 Jahren ein bundesweites, kostenfreies und anonymes Gesprächs- und Beratungsangebot für Kinder und Jugendliche. Für die Beratungsarbeit am KJT werden interessierte und sozial engagierte Frauen und Männer sorgfältig ausgewählt und umfassend ausgebildet. Die Beraterinnen und Berater sind ausschließlich ehrenamtlich tätig. Eine Weiterentwicklung der „herkömmlichen“ Telefonberatung stellte das im Jahr 1998 ins Leben gerufene Angebot „Jugendliche 
beraten Jugendliche“ ( JbJ) dar. Hier werden Jugendliche im Alter von 16-21 Jahren für die telefonische Beratung am KJT eingesetzt. Die Idee dabei ist es, Gleichaltrige als Ansprechpartner bei Fragen und Problemen speziell des Jugendalters in Anspruch nehmen zu können. In ihrem Beitrag über den Personzentrierten Ansatz in der Ausbildung jugendlicher Berater für das Kinder- und Jugendtelefon (KJT) stellt Susann Pruchnik Forschungsergebnisse und ihre eigenen Erfahrungen als Beraterin und Ausbilderin dar.

Vor allem im psychosozialen Bereich hat sich der Personzentrierte Ansatz bewährt. In Wirtschaftsunternehmen ist dieser Ansatz meist unbekannt und nicht gewollt oder nicht realisierbar. In dem Beitrag von Björn Seidel wird jedoch gezeigt, dass es auch in einem derartigen Umfeld Bereiche gibt, in denen der Personzentrierte Ansatz integrierbar ist. Seidel geht es um Potenzialanalysen als strategisches Human Resources Management und um die Frage der Integrierbarkeit des personzentrierten Ansatzes. Potenzialanalysen werden meist durchgeführt, um herauszufinden, ob Mitarbeiter innerhalb des Unternehmens für weitere Aufgaben oder Positionen geeignet sind oder um bei Neueinstellungen die Bewerber zu identifizieren, die das größte Potenzial aufweisen, um erfolgreich für das Unternehmen tätig zu sein. Unter personzentrierten Aspekten sind Potenzialanalysen zunächst kritisch zu betrachten, da das Beurteilen und Bewerten von Menschen dem Ansatz Rogers' entgegensteht. Seidel kommt jedoch zu dem Ergebnis, dass - abhängig vom eingesetzten Potenzialanalyseverfahren - der Personzentrierte Ansatz auch in einem derartigen Umfeld realisierbar und integrierbar ist. Um dies zu ermöglichen, ist jedoch neben entsprechend ausgebildeten Unternehmensberatern auch eine Weiterentwicklung und eine Öffnung des Personzentrierten Ansatzes notwendig.

Die psychotherapeutische und beraterische Betreuung von Personen, die als Opfer, Zeugen oder aus beruflichen Gründen (Polizei, Rettungsdienste usw.) in Katastrophen involviert sind, hat in den letzten Jahren erheblich an Bedeutung gewonnen. Eine wichtige Frage ist die, wie den betroffenen Personen am besten geholfen werden kann. Holger Reiprich, Theologe und Unfallseelsorger, vergleicht in seinem Beitrag das ,, Critical Incident Stress Management“ und die „Personzentrierte Krisenintervention “. Das Ergebnis ist bemerkenswert, denn das Critical Incident Stress Management ist zwar in Deutschland weit verbreitet, weist jedoch einige Schwächen auf, die - wenigstens zum Teil - durch einen personzentrierten Ansatz überwunden werden können.

Nicht nur diese letzte Arbeit zeigt die Notwendigkeit und den Nutzen systematischer Forschung im Beratungsbereich auf. So verbinden wir mit der Veröffentlichung dieser Arbeiten den Wunsch, dass Personzentrierte Beratung weitere Verbreitung findet und häufiger Inhalt systematischer empirischer Forschung im Interesse der Praxis wird.

Der Thementeil dieses Heftes wird um Rezensionen einiger neuer Bücher zur Beratung und personzentrierten Psychotherapie abgerundet.

Wir wünschen gute Lektüre! 